Operations Research and Applications : An International Journal (ORAJ), Vol.5, No.2, May 2018

\title{
A NEW ALGORITHM FOR SOLVING FULLY FUZZY BI-LEVEL QUADRATIC PROGRAMMING PROBLEMS
}

\author{
Azza H. Amer \\ Department of Mathematics, Faculty of Science, \\ Helwan University, Cairo, Egypt
}

\begin{abstract}
This paper is concerned with new method to find the fuzzy optimal solution of fully fuzzy bi-level non-linear (quadratic) programming (FFBLQP) problems where all the coefficients and decision variables of both objective functions and the constraints are triangular fuzzy numbers $\left(T F N_{s}\right)$. A new method is based on decomposed the given problem into bi-level problem with three crisp quadratic objective functions and bounded variables constraints. In order to often a fuzzy optimal solution of the FFBLQP problems, the concept of tolerance membership function is used to develop a fuzzy max-min decision model for generating satisfactory fuzzy solution for FFBLQP problems in which the upper-level decision maker (ULDM) specifies his/her objective functions and decisions with possible tolerances which are described by membership functions of fuzzy set theory. Then, the lower-level decision maker (LLDM) uses this preference information for ULDM and solves his/her problem subject to the ULDM restrictions. Finally, the decomposed method is illustrated by numerical example.
\end{abstract}

\section{KEYWORDS}

Fully Fuzzy Quadratic Non-Linear Programming Problems. Triangular Fuzzy Numbers, Decomposed Method, Bi-Level Programming.

\section{INTRODUCTION}

The bi-level programming (BLP) problem is considered a useful optimization problems in which there are independent decision makers $\left(\mathrm{DM}_{\mathrm{s}}\right)$ and the feasible region of the upper-level (UL) problem is determined implicitly by the solution set of the lower-level (LL) problem. In the past few decades, the BLP problem has been covered the theoretical and computational points [1-11] and has been applied indifferent fields such as finance budget, transport network design [12], supply chain management [13], principal-agent problem [14] engineering design [15], price control and electricity markets.

In recent decades, the bi-level decision making problems became very hard to find the values of the coefficients because of imprecise information when finding these models. So, fuzzy set theory has been applied to handle imprecise data [16] where the coefficients in both objective functions and the constraints are described by fuzzy numbers. 
Operations Research and Applications : An International Journal (ORAJ), Vol.5, No.2, May 2018

Tanaka et al. [17] was first proposed the concept of fuzzy mathematical programming (FMP) on general level in the framework of the fuzzy decision of Bellman and Zadeh [18]. Zimmermann [19] was first proposed the formulation of fuzzy linear programming. Also, Sakawa et al. [20-23] first formulated the fuzzy bi-level programming problem and developed a fuzzy programming method to solve it. Many researchers adopted this concept for solving FLP problems. It is notice that the decision variables of all the above works are not fuzzy and the crisp solutions are ineligible to describe the fuzzy advantage of the decision making process in an uncertain environment.

In recent years, the fully fuzzy linear programming (FFLP) problems in which the coefficients as well as decision variables are described by fuzzy numbers has been an attractive topic for the researchers. Liou and Wang [24] proposed the concepts of ranking fuzzy numbers which is playing a very important role in decision making. Also, there are a number of researchers who have developed and presented new methods in this field of FFLP such as [25-32]. For the fully fuzzy non-linear programming problems, Walaa Ibrahim Gabr [33] presents a comprehensive methodology for solving and analyzing Quadratic and non-linear programming problems in fully fuzzy environment. It should be noted that all these works are considered in the case of onesingle-level FFLP.

To our knowledge, until now there are few researcher studies the type of fully fuzzy bi-level linear programming (FFBLLP) problem in which all coefficients and variable of both objective functions and the constraints are expressed as fuzzy number such as [34-37].

The aim of this paper is to develop a new method to deal with the fully fuzzy bi-level quadratic programming problems by applying the concept of tolerance membership function to show that, the satisfactory solution obtained by fuzzy max-min decision model are always fuzzy optimal solution $[38,39]$. A numerical example is given to illustrate the proposed method.

\section{PRELIMINARIES}

In this section, some basic definitions of the fuzzy number and fuzzy arithmetic operations depending on fuzzy numbers are reviewed.

\section{Definition 1[38]}

The characteristic function $\mu_{A}$ of a crisp set $A \subseteq X$ assigns a value either 0 or 1 to each member in $X$. This function can be generalized to a function $\mu_{\tilde{A}}$ such that the value assigned to the element of the universal set $\mathrm{X}$ fall within a specified range i.e. $\mu_{\tilde{A}}: X \rightarrow[0,1]$. The assigned value indicate the membership grade of the element in the set $A$. The function $\mu_{\tilde{A}}$ is called the membership function and the set $A=\left\{\left(x, \mu_{\tilde{A}}(x)\right): x \in X\right\}$ defined by $\mu_{\tilde{A}}(x)$ for each $x \in X$ is called a fuzzy set.

\section{Definition 2[38]}

A fuzzy number $\tilde{A}=(a, b, c)$ is said to be a triangular fuzzy number if its membership function is given by 
Operations Research and Applications : An International Journal (ORAJ), Vol.5, No.2, May 2018

$$
\mu_{\tilde{A}}(x)=\left\{\begin{array}{cc}
\frac{x-a}{b-a}, & a \leq x \leq b, \\
1, & x=b, \\
\frac{x-c}{b-c}, & b \leq x \leq c .
\end{array}\right.
$$

\section{Definition 3[38]}

A triangular fuzzy number (a, b, c) is said to be non-negative fuzzy number iff $a \geq 0$.

Definition 4[38] (Arithmetic Operations)

Let $\tilde{A}=(a, b, c)$ and $\tilde{B}=(e, f, g)$ be two triangular fuzzy numbers. The algebraic operations between any two triangular fuzzy numbers $\tilde{A}$ and $\widetilde{B}$ can be defined by:

1) $\tilde{A} \oplus \tilde{B}=(a+e, b+f, c+g)$

2) $-\tilde{A}=-(a, b, c)=(-c,-b,-a)$

3) $\tilde{A} \Theta \widetilde{B}=(a-g, b-f, c-e)$

4) $k \tilde{A}= \begin{cases}(k a, k b, k c) & , k \geq 0, \\ (k c, k b, k a) & , k<0\end{cases}$

5) Let $\tilde{A}=(a, b, c)$ be an arbitrary triangular fuzzy number and let $\widetilde{B}=(e, f, g)$ be a nonnegative triangular fuzzy number, then

$$
\tilde{A} \otimes \tilde{B}=\left\{\begin{array}{cc}
(a e, b f, c g) & , \quad a \geq 0, \\
(a g, b f, c g) & , \quad a<0, c \geq 0, \\
(a g, b f, c e) & , \quad c<0 .
\end{array}\right.
$$

\section{FORMULATION OF THE FULLY FUZZY BI-LEVEL QUADRATIC Programming Problem}

Consider the following FFBLQP problem in which all the coefficients and the decision variables are fuzzy numbers:

\section{FFULDM:}

$$
\max _{\tilde{x}_{1}} \tilde{F}_{1}\left(\tilde{x}_{1}, \tilde{x}_{2}\right)=\max _{\tilde{x}_{1}}\left(\tilde{c}_{11} \otimes \tilde{x}_{1}^{2} \oplus \tilde{c}_{12} \otimes \tilde{x}_{2}^{2}\right)
$$

where $\tilde{x}_{2}$ solves

\section{FFLLDM:}

$$
\max _{\tilde{x}_{2}} \tilde{F}_{2}\left(\tilde{x}_{1}, \tilde{x}_{2}\right)=\max _{\tilde{x}_{2}}\left(\tilde{c}_{21} \otimes \tilde{x}_{1}^{2} \oplus \tilde{c}_{22} \otimes \tilde{x}_{2}^{2}\right)
$$

s.t. 
Operations Research and Applications : An International Journal (ORAJ), Vol.5, No.2, May 2018

$$
\begin{gathered}
\tilde{G}=\left\{\left(\tilde{x}_{1}, \tilde{x}_{2}\right) \mid \tilde{a}_{i 1} \otimes \tilde{x}_{1} \oplus \tilde{a}_{i 2} \otimes \tilde{x}_{2} \leq \tilde{b}_{i}, i=1,2, \ldots, m,\right. \\
\left.\tilde{x}_{1} \geq \tilde{0}, \tilde{x}_{2} \geq \tilde{0}\right\} .
\end{gathered}
$$

where $\tilde{x}_{1}=\left(\tilde{x}_{11}, \tilde{x}_{12}, \ldots, \tilde{x}_{1 n_{1}}\right) \in R^{n_{1}}$ is an $n_{1}$-dimensional fuzzy decision vector of the upper-level, and $\tilde{x}_{2}=\left(\tilde{x}_{21}, \tilde{x}_{22}, \ldots, \tilde{x}_{2 n_{2}}\right) \in R^{n_{2}}$ is an $n_{2}$-dimensional fuzzy decision vector of the lower-level.

Let $\tilde{F}_{1}: R^{n_{1}} \times R^{n_{2}} \rightarrow R^{N_{1}}$ be the upper-level objective functions and $\tilde{F}_{2}: R^{n_{1}} \times R^{n_{2}} \rightarrow R^{N_{2}}$ be the lower-level objective functions, $N_{1}, N_{2} \geq 2$, elements $\tilde{x}_{j k_{j}}, j=1,2, k_{j}=1,2, \ldots, n_{j}$ of decision vectors $\tilde{x}_{j}$ are non-negative fuzzy triangular fuzzy numbers; $\tilde{c}_{L j}=\left(\tilde{c}_{L j_{1}}, \tilde{c}_{L j_{2}}, \ldots, \tilde{c}_{L j_{n j}}\right)$, $L=1,2, j=1,2$ and $\tilde{a}_{i j}=\left(\tilde{a}_{i j_{1}}, \tilde{a}_{i j_{2}}, \ldots, \tilde{a}_{i j n_{j}}\right), i=1,2, \ldots, m$, are $n_{j}$-dimensional fuzzy vectors; elements $\tilde{c}_{L j_{s}}, \tilde{a}_{i j_{t}}, s, t=1,2, \ldots, n_{j}$ and $\tilde{b}_{i}$ are fuzzy numbers; $\tilde{G}$ is the constraint region of problem (1)-(3).

\subsection{Decomposition Method of FFBLQ P problem.}

In this section, a new method is proposed to find the fuzzy optimal solution of FFBLQP problem. In this way, one first gets the fuzzy satisfactory solution is acceptable to FFULDM, then FFULDM gives fuzzy decision variables and fuzzy goals with some information to the FFLLDM for him/her to seek the fuzzy satisfactory solution and to arrive at the fuzzy solution which is closest to the fuzzy satisfactory solution of the FFULDM. This due to, the FFLLDM should not only optimize his/her objective functions but also try to satisfy the FFULDM's goals and preference as much as possible [1].

Let the fuzzy parameters $\tilde{F}_{j}, \tilde{x}_{j}, c_{L j}, a_{i j}$ and $b_{i}, j=1,2, L=1,2, i=1,2, \ldots, m$ be the triangular fuzzy numbers which is represented by $\left(0^{L}, 0^{m}, 0^{u}\right)$, then problem (1) - (3) can be written as:

\section{FFULDM:}

$$
\begin{gathered}
\max _{\left(x_{1 k_{1}}^{L}, x_{1 k}^{m}, x_{1 k_{1}}^{u}\right)}\left(F_{1}^{L}, F_{1}^{m}, F_{1}^{u}\right)=\max _{\left(x_{1 k_{1}, k k_{1},}^{L}, x_{1 k_{1}}^{u}\right)}\left(\sum_{k_{1}=1}^{n_{1}}\left(c_{11 k_{1}}^{L}, c_{11 k_{1}}^{m}, c_{11 k_{1}}^{u}\right) \otimes\left(x_{1 k_{1}}^{2 L}, x_{1 k_{1}}^{2 m}, x_{1 k 1}^{2 u}\right)\right. \\
\left.\oplus \sum_{k_{2}=1}^{n_{2}}\left(c_{12 k_{2}}^{L}, c_{12 k_{2}}^{m}, c_{12 k_{2}}^{u}\right) \otimes\left(x_{2 k_{2}}^{2 L}, x_{2 k_{2}}^{2 m}, x_{2 k_{2}}^{2 u}\right)\right),
\end{gathered}
$$

where $\left(x_{2}^{L}, x_{2}^{m}, x_{2}^{u}\right)$ solves

\section{FFLLDM:}

$$
\begin{gathered}
\max _{\left(x_{2 k_{2}}^{L}, x_{2 k_{2}}^{m}, x_{2 k_{2}}^{u}\right)}\left(F_{2}^{L}, F_{2}^{m}, F_{2}^{u}\right)=\max _{\left(x_{2 k_{2}}^{L}, x_{2 k_{2}}^{m}, x_{2 k_{2}}^{u}\right)}\left(\sum_{k_{1}=1}^{n_{1}}\left(c_{21 k_{1}}^{L}, c_{21 k_{1}}^{m}, c_{21 k_{1}}^{u}\right) \otimes\left(x_{1 k_{1}}^{2 L}, x_{1 k_{1}}^{2 m}, x_{1 k_{1}}^{2 u}\right)\right. \\
\left.\oplus \sum_{k_{2}=1}^{n_{2}}\left(c_{22 k_{2}}^{L}, c_{22 k_{2}}^{m}, c_{22 k_{2}}^{u}\right) \otimes\left(x_{2 k_{2}}^{2 L}, x_{2 k_{2}}^{2 m}, x_{2 k_{2}}^{2 u}\right)\right),
\end{gathered}
$$


Operations Research and Applications : An International Journal (ORAJ), Vol.5, No.2, May 2018

$$
\begin{aligned}
& \text { s.t. } \\
& G=\left\{\sum_{k_{1}=1}^{n_{1}}\left(a_{1 i_{k_{1}}}^{L}, a_{1 i_{k_{1}}}^{m}, a_{1 i_{k_{1}}}^{u}\right) \otimes\left(x_{1 k_{1}}^{L}, x_{1 k_{1}}^{m}, x_{1 k_{1}}^{u}\right) \oplus \sum_{k_{2}=1}^{n_{2}}\left(a_{2 i_{k_{2}}}^{L}, a_{2 i_{k_{2}}}^{m}, a_{2 i_{k_{2}}}^{u}\right) \otimes\left(x_{2 k_{2}}^{L}, x_{2 k_{2}}^{m}, x_{2 k_{2}}^{u}\right)\right. \\
& \leq\left(b_{i}^{L}, b_{i}^{m}, b_{i}^{u}\right), \quad i=1,2, \ldots, m, \\
& \left.\left(x_{1}^{L}, x_{1}^{m}, x_{1}^{u}\right) \geq(0,0,0),\left(x_{2}^{L}, x_{2}^{m}, x_{2}^{u}\right) \geq(0,0,0)\right\}
\end{aligned}
$$

Now, using the arithmetic operations (see def. 4), then problem (4)-(6) can be decomposed into three crisp bi-level quadratic programming (BLQP) problem as:

\section{FULDM:}

$$
\begin{aligned}
& \max _{x_{1 k_{1}}} F_{1}^{L}=\text { lower value of }\left[\sum_{k_{1}=1}^{n_{1}}\left(c_{11 k_{1}}^{L}, c_{11 k_{1}}^{m}, c_{11 k_{1}}^{u}\right) \otimes\left(x_{1 k_{1}}^{2 L}, x_{1 k_{1}}^{2 m}, x_{1 k_{1}}^{2 u}\right)\right. \\
&\left.\oplus \sum_{k_{2}=1}^{n_{2}}\left(c_{12 k_{2}}^{L}, c_{12 k_{2}}^{m}, c_{12 k_{2}}^{u}\right) \otimes\left(x_{2 k_{2}}^{2 L}, x_{2 k_{2}}^{2 m}, x_{2 k_{2}}^{2 u}\right)\right], \\
& \max _{x_{1 k_{1}}} F_{1}^{m}=\operatorname{middle~value~of~}\left[\sum_{k_{1}=1}^{n_{1}}\left(c_{11 k_{1}}^{L}, c_{11 k_{1}}^{m}, c_{11 k_{1}}^{u}\right) \otimes\left(x_{1 k_{1}}^{2 L}, x_{1 k_{1}}^{2 m}, x_{1 k_{1}}^{2 u}\right)\right. \\
&\left.\oplus \sum_{k_{2}=1}^{n_{2}}\left(c_{12 k_{2}}^{L}, c_{12 k_{2}}^{m}, c_{12 k_{2}}^{u}\right) \otimes\left(x_{2 k_{2}}^{2 L}, x_{2 k_{2}}^{2 m}, x_{2 k_{2}}^{2 u}\right)\right], \\
& \max _{x_{1 k_{1}}} F_{1}^{u}=\operatorname{upper} \text { value of }\left[\sum_{k_{1}=1}^{n_{1}}\left(c_{11 k_{1}}^{L}, c_{11 k_{1}}^{m}, c_{11 k_{1}}^{u}\right) \otimes\left(x_{1 k_{1}}^{2 L}, x_{1 k_{1}}^{2 m}, x_{1 k_{1}}^{2 u}\right)\right. \\
&\left.\oplus \sum_{k_{2}=1}^{n_{2}}\left(c_{12 k_{2}}^{L}, c_{12 k_{2}}^{m}, c_{12 k_{2}}^{u}\right) \otimes\left(x_{2 k_{2}}^{2 L}, x_{2 k_{2}}^{2 m}, x_{2 k_{2}}^{2 u}\right)\right],
\end{aligned}
$$

where $\left(x_{2}^{L}, x_{2}^{m}, x_{2}^{u}\right)$ solves

\section{FLLDM:}

$$
\begin{aligned}
& \max _{x_{2 k_{2}}} F_{2}^{L}=\text { lower value of }\left[\sum_{k_{1}=1}^{n_{1}}\left(c_{21 k_{1}}^{L}, c_{21 k_{1}}^{m}, c_{21 k_{1}}^{u}\right) \otimes\left(x_{1 k_{1}}^{2 L}, x_{1 k_{1}}^{2 m}, x_{1 k_{1}}^{2 u}\right)\right. \\
&\left.\oplus \sum_{k_{2}=1}^{n_{2}}\left(c_{22 k_{2}}^{L}, c_{22 k_{2}}^{m}, c_{22 k_{2}}^{u}\right) \otimes\left(x_{2 k_{2}}^{2 L}, x_{2 k_{2}}^{2 m}, x_{2 k_{2}}^{2 u}\right)\right], \\
& \max _{x_{2 k_{2}}} F_{2}^{m}= \text { middle value of }\left[\sum_{k_{1}=1}^{n_{1}}\left(c_{21 k_{1}}^{L}, c_{21 k_{1}}^{m}, c_{21 k_{1}}^{u}\right) \otimes\left(x_{1 k_{1}}^{2 L}, x_{1 k_{1}}^{2 m}, x_{1 k_{1}}^{2 u}\right)\right. \\
&\left.\oplus \sum_{k_{2}=1}^{n_{2}}\left(c_{22 k_{2}}^{L}, c_{22 k_{2}}^{m}, c_{22 k_{2}}^{u}\right) \otimes\left(x_{2 k_{2}}^{2 L}, x_{2 k_{2}}^{2 m}, x_{2 k_{2}}^{2 u}\right)\right]
\end{aligned}
$$


Operations Research and Applications : An International Journal (ORAJ), Vol.5, No.2, May 2018

$$
\begin{aligned}
\max _{x_{2 k_{2}}} F_{2}^{u} & =\text { upper value of }\left[\sum_{k_{1}=1}^{n_{1}}\left(c_{21 k_{1}}^{L}, c_{21 k_{1}}^{m}, c_{21 k_{1}}^{u}\right) \otimes\left(x_{1 k_{1}}^{2 L}, x_{1 k_{1}}^{2 m}, x_{1 k_{1}}^{2 u}\right)\right. \\
& \left.\oplus \sum_{k_{2}=1}^{n_{2}}\left(c_{22 k_{2}}^{L}, c_{22 k_{2}}^{m}, c_{22 k_{2}}^{u}\right) \otimes\left(x_{2 k_{2}}^{2 L}, x_{2 k_{2}}^{2 m}, x_{2 k_{2}}^{2 u}\right)\right],
\end{aligned}
$$

s.t.

$$
\begin{aligned}
& G=\left\{\text { lower value of } \left[\sum_{k_{1}=1}^{n_{1}}\left(a_{1 i_{k_{1}}}^{L}, a_{1 i_{k_{1}}}^{m}, a_{1 i_{k_{1}}}^{u}\right) \otimes\left(x_{1 k_{1}}^{L}, x_{1 k_{1}}^{m}, x_{1 k_{1}}^{u}\right)\right.\right. \\
& \left.\oplus \sum_{k_{2}=1}^{n_{2}}\left(a_{2 i_{k_{2}}}^{L}, a_{2 i_{k_{2}}}^{m}, a_{2 i_{k_{2}}}^{u}\right) \otimes\left(x_{2 k_{2}}^{L}, x_{2 k_{2}}^{m}, x_{2 k_{2}}^{u}\right)\right] \leq b_{i}^{L}, i=1,2, \ldots, m,
\end{aligned}
$$

middle value of $\left[\sum_{k_{1}=1}^{n_{1}}\left(a_{1 i_{k_{1}}}^{L}, a_{1 i_{k_{1}}}^{m}, a_{1 k_{k_{1}}}^{u}\right) \otimes\left(x_{1 k_{1}}^{L}, x_{1 k_{1}}^{m}, x_{1 k_{1}}^{u}\right)\right.$

$$
\left.\oplus \sum_{k_{2}=1}^{n_{2}}\left(a_{2 i_{k_{2}}}^{L}, a_{2 i_{k_{2}}}^{m}, a_{2 i_{k_{2}}}^{u}\right) \otimes\left(x_{2 k_{2}}^{L}, x_{2 k_{2}}^{m}, x_{2 k_{2}}^{u}\right)\right] \leq b_{i}^{m}, i=1,2, \ldots, m,
$$

upper value of $\left[\sum_{k_{1}=1}^{n_{1}}\left(a_{1 k_{k_{1}}}^{L}, a_{1 i_{k_{1}}}^{m}, a_{1 i_{k_{1}}}^{u}\right) \otimes\left(x_{1 k_{1}}^{L}, x_{1 k_{1}}^{m}, x_{1 k_{1}}^{u}\right)\right.$

$$
\begin{aligned}
& \left.\oplus \sum_{k_{2}=1}^{n_{2}}\left(a_{2 i_{k_{2}}}^{L}, a_{2 i_{k_{2}}}^{m}, a_{2 i_{k_{2}}}^{u}\right) \otimes\left(x_{2 k_{2}}^{L}, x_{2 k_{2}}^{m}, x_{2 k_{2}}^{u}\right)\right] \leq b_{i}^{u}, i=1,2, \ldots, m, \\
& \left.x_{j k_{j}}^{m}-x_{j k_{j}}^{L} \geq 0, x_{j k_{j}}^{u}-x_{j k_{j}}^{m} \geq 0, j=1,2, k=1,2, \ldots, m\right\}
\end{aligned}
$$

\section{Definition 5 [2]}

For any $\tilde{x}_{1}\left(\tilde{x}_{1} \in \tilde{G}_{0}=\left\{\left(\tilde{x}_{1}, \tilde{x}_{2}\right) \in G\right\}\right)$ given by FULDM, if the fuzzy decision making variable $\tilde{x}_{2}\left(\tilde{x}_{2} \in \tilde{G}_{1}=\left\{\left(\tilde{x}_{1}, \tilde{x}_{2}\right) \in G\right\}\right)$ at the lower- level (LL) is the fuzzy optimal solution of FLLDM, then $\left(\tilde{x}_{1}, \tilde{x}_{2}\right)$ is a fuzzy feasible solution of problem (1) - (3).

\section{Definition 6 [2]}

$\left(\tilde{x}_{1}^{*}, \tilde{x}_{2}^{*}\right)$ is a fuzzy feasible solution of FFBLQP problem (1)-(3); no other fuzzy feasible solution $\left(\tilde{x}_{1}, \tilde{x}_{2}\right) \in \tilde{G}$ exists, such that $\tilde{f}_{1 s}\left(\tilde{x}_{1}^{*}, \tilde{x}_{2}^{*}\right) \leq \tilde{f}_{1 s}\left(\tilde{x}_{1}, \tilde{x}_{2}\right)$; at least one $\mathrm{s}\left(\mathrm{s}=1,2, \ldots, \mathrm{N}_{1}\right)$ is strict inequality, then $\left(\tilde{x}_{1}^{*}, \tilde{x}_{2}^{*}\right)$ is the fuzzy optimal solution of problem (1)-(3).

\subsection{Fuzzy Decision Models for BLQP Problem}

In this section, the solution method simplifies a BLQP problem by transforming it into separate quadratic decision making (QDM) problems at upper and lower- levels as: 
Operations Research and Applications : An International Journal (ORAJ), Vol.5, No.2, May 2018

\subsubsection{FULDM Problem}

The FULDM solves the following fuzzy multi-objective decision making (FMODM) problem as:

$$
\begin{aligned}
& \max _{\tilde{x}} \tilde{F}_{1}(\tilde{x})=\max _{\tilde{x}}\left(F_{1}^{L}(\tilde{x}), F_{1}^{m}(\tilde{x}), F_{1}^{u}(\tilde{x})\right) \\
& \text { s.t. } \\
& \tilde{x} \in G
\end{aligned}
$$

where $\tilde{x}=\left(\tilde{x}_{1}, \tilde{x}_{2}\right), \tilde{x} \in R^{n_{1}+n_{2}}$

We should first find individual best fuzzy solutions $\tilde{F}_{1}^{+}$and individual worst fuzzy solutions $\widetilde{F}_{1}^{-}$for each objective of (10), where [4]:

$$
\tilde{F}_{1}^{+}=\max _{\tilde{x} \in G} F_{1}(\tilde{x}) \text { and } \tilde{F}_{1}^{-}=\min _{\tilde{x} \in G} F_{1}(\tilde{x})
$$

Goals and tolerances can then be reasonably set for individual best fuzzy solutions and the differences of the best and worst fuzzy solutions, respectively. This data can then be formulated as the following membership functions of fuzzy set theory $[18,19]$ as:

$$
\mu\left[\tilde{F}_{1}(\tilde{x})\right]=\left\{\begin{array}{cl}
1 & , \quad \text { if } \tilde{F}_{1}(\tilde{x})>\tilde{F}_{1}^{+} \\
\frac{\tilde{F}_{1}(\tilde{x})-\tilde{F}_{1}^{-}}{\widetilde{F}_{1}^{+}-\tilde{F}_{1}^{-}}, & \text {if } \tilde{F}_{1}^{-} \leq \tilde{F}_{1}(\tilde{x}) \leq \tilde{F}_{1}^{+}, \\
0 & , \text { if } \tilde{F}_{1}^{-} \geq \tilde{F}_{1}(\tilde{x})
\end{array}\right.
$$

Also, we can find the fuzzy solution of the FULDM problem by solving the following Tchebycheff problem $[18,19]$ as:

$$
\begin{aligned}
& \max \tilde{\lambda}_{1} \\
& \text { s.t. } \\
& \tilde{x} \in G, \\
& \mu\left[\tilde{F}_{1}(\tilde{x})\right] \geq \tilde{\lambda}_{1}, \quad \tilde{\lambda}_{1} \in[0,1] .
\end{aligned}
$$

where $\tilde{\lambda}_{1}=\left(\tilde{\lambda}_{1}^{L}, \tilde{\lambda}_{1}^{m}, \tilde{\lambda}_{1}^{u}\right)$ is satisfactory level and the fuzzy solution is assumed to be $\left(\tilde{x}_{1}^{u}, \tilde{x}_{2}^{u}, \tilde{F}_{1}^{u}\right.$ and $\left.\tilde{\lambda}_{1}^{u}\right)$ for the upper-level.

\subsubsection{FLLDM Problem}

In the same way, the FLLDM independently solves:

$$
\begin{aligned}
& \max _{\tilde{x}} \widetilde{F}_{2}(\tilde{x})=\max _{\tilde{x}}\left(F_{2}^{L}(\tilde{x}), F_{2}^{m}(\tilde{x}), F_{2}^{u}(\tilde{x})\right) \\
& \text { s.t. } \\
& \tilde{x} \in G,
\end{aligned}
$$$$
\text { where } \tilde{x}=\left(\tilde{x}_{1}, \tilde{x}_{2}\right), \quad \tilde{x} \in R^{n_{1}+n_{2}}
$$

The individual best fuzzy solutions $\tilde{F}_{2}^{+}$and individual worst fuzzy solutions $\tilde{F}_{2}^{-}$for each objective of (14) are: 
Operations Research and Applications : An International Journal (ORAJ), Vol.5, No.2, May 2018

$$
\tilde{F}_{2}^{+}=\max _{\tilde{x} \in G} F_{2}(\tilde{x}) \text { and } \tilde{F}_{2}^{-}=\min _{\tilde{x} \in G} F_{2}(\tilde{x})
$$

From this information, the membership functions can be formulated by using fuzzy theorem as:

$$
\mu\left[\tilde{F}_{2}(\tilde{x})\right]=\left\{\begin{array}{cl}
1, & \text { if } \tilde{F}_{2}(\tilde{x})>\tilde{F}_{2}^{+} \\
\frac{\tilde{F}_{2}(\bar{x})-\tilde{F}_{2}^{-}}{\widetilde{F}_{2}^{+}-\tilde{F}_{2}^{-}}, & \text {if } \tilde{F}_{2}^{-} \leq \tilde{F}_{2}(\bar{x}) \leq \tilde{F}_{2}^{+}, \\
0 & , \text { if } \tilde{F}_{2}^{-} \geq \tilde{F}_{2}(\tilde{x})
\end{array} .\right.
$$

Now, we can obtain the fuzzy solution of the FLLDM problem by solving the following Tchebycheff problem as [18, 19]:

$$
\begin{aligned}
& \max \tilde{\lambda}_{2} \\
& \text { s.t. } \\
& \tilde{x} \in G, \\
& \mu\left[\tilde{F}_{2}(\tilde{x})\right] \geq \tilde{\lambda}_{2}, \tilde{\lambda}_{2} \in[0,1] .
\end{aligned}
$$

Whose fuzzy solution is assumed to be $\left(\tilde{x}_{1}^{L}, \tilde{x}_{2}^{L}, \tilde{F}_{2}^{L}\right.$ and $\left.\tilde{\lambda}_{2}^{L}\right)$ for the lower-level and $\tilde{\lambda}_{2}^{L}$ is satisfactory level.

\subsubsection{FFBLQDM Problem}

Finally, in order to generate the fuzzy satisfactory solution which is also a fuzzy optimal solution with an overall satisfaction for both $\mathrm{DM}_{\mathrm{s}}$, we can solve the following Tchebycheff problem [4, 18, 19] as:

$$
\begin{aligned}
& \max \tilde{\lambda} \\
& \text { s.t. } \\
& \tilde{x} \in G, \\
& \quad\left[\left(\tilde{x}_{1}^{u}+\tilde{t}_{1}\right)\right]-\tilde{x} / \tilde{t}_{1} \geq \tilde{\lambda} I \\
& \quad\left[\tilde{x}_{1}-\left(\tilde{x}_{1}^{u}-\tilde{t}_{1}\right)\right] / \tilde{t}_{1} \geq \tilde{\lambda} I, \\
& \quad \mu_{\tilde{F}_{1}}^{\prime}\left[\tilde{F}_{1}(\tilde{x})\right]=\left[\tilde{F}_{1}(\tilde{x})-\tilde{F}_{1}^{\prime}\right] /\left[\tilde{F}_{1}^{u}-\tilde{F}_{1}^{\prime}\right] \geq \tilde{\lambda}, \\
& \quad \mu_{\tilde{F}_{2}}^{\prime}\left[\tilde{F}_{2}(\tilde{x})\right]=\left[\tilde{F}_{2}(\tilde{x})-\tilde{F}_{2}^{\prime}\right] /\left[\tilde{F}_{2}^{L}-\tilde{F}_{2}^{\prime}\right] \geq \tilde{\lambda}, \\
& \quad \tilde{x}_{1} \geq \tilde{0}, \quad \tilde{x}_{2} \geq \tilde{0} \text { and } \tilde{\lambda} \in[0,1] .
\end{aligned}
$$

where $\tilde{\lambda}$ is the overall satisfaction, $\mathrm{I}$ is the column vector with all elements equal to $1_{\mathrm{s}}$ and the same dimension as $\tilde{x}_{1} ; \tilde{x}_{1}^{u}$ is the fuzzy optimal decision variable of the FULDM, $\tilde{t}_{1}$ is maximum tolerances of the range of the decision variable $\tilde{x}_{1}$ around $\tilde{x}_{1}^{u}$ and the membership function of $\tilde{x}_{1}$ can be stated as follows: 
Operations Research and Applications : An International Journal (ORAJ), Vol.5, No.2, May 2018

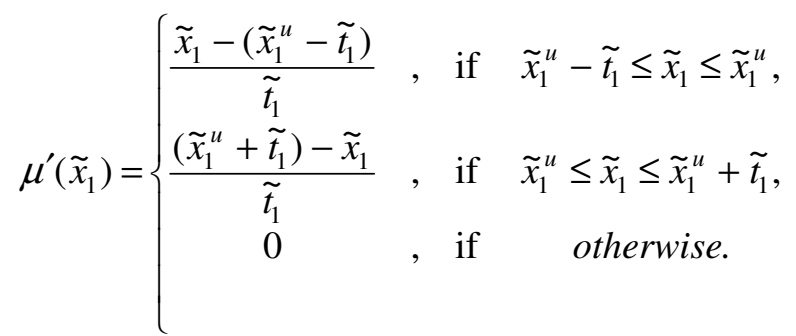

The following membership functions of the FULDM can be stated as:

$$
\mu_{\tilde{F}_{1}}^{\prime}\left[\tilde{F}_{1}(\tilde{x})\right]=\left\{\begin{array}{cl}
1 & , \text { if } \tilde{F}_{1}(\tilde{x})>\tilde{F}_{1}^{u}, \\
\frac{\tilde{F}_{1}(\tilde{x})-\tilde{F}_{1}^{\prime}}{\tilde{F}_{1}^{u}-\tilde{F}_{1}^{\prime}}, & \text { if } \tilde{F}_{1}^{\prime} \leq \tilde{F}_{1}(\bar{x}) \leq \tilde{F}_{1}^{u}, \\
0 & , \text { if } \tilde{F}_{1}(\tilde{x}) \leq \tilde{F}_{1}^{\prime}
\end{array} .\right.
$$

where $\tilde{F}_{1}^{\prime}=\tilde{F}_{1}\left(x_{1}^{L}, x_{2}^{L}\right)$.

Also, the FLLDM has the following membership functions for his/her goals as:

$$
\mu_{\tilde{F}_{2}}^{\prime}\left[\tilde{F}_{2}(\tilde{x})\right]=\left\{\begin{array}{cl}
1, & \text { if } \tilde{F}_{2}(\tilde{x})>\tilde{F}_{2}^{L} \\
\frac{\tilde{F}_{2}(\tilde{x})-\tilde{F}_{2}^{\prime}}{\tilde{F}_{2}^{u}-\tilde{F}_{2}^{\prime}}, & \text { if } \tilde{F}_{2}^{\prime} \leq \tilde{F}_{2}(\tilde{x}) \leq \tilde{F}_{2}^{L}, \\
0 & , \text { if } \tilde{F}_{2}(\tilde{x}) \leq \tilde{F}_{2}^{\prime}
\end{array}\right.
$$

where $\tilde{F}_{2}^{\prime}=\tilde{F}_{2}\left(x_{1}^{u}, x_{2}^{u}\right)$.

By solving problem (18) for three decomposition problems, if the FULDM is satisfied with this solution, then a fuzzy satisfactory solution is reached. Otherwise, he/she should provide new membership functions for the fuzzy control variable and objectives to FLLDM until a fuzzy satisfactory solution is reached.

\section{A NEW AlgorithM FOR SOLVING FFBLQP PROBLEM}

The steps for the computation of FFBLQP problem can be summarized as:

Step 1: Consider the variables and parameters of FFBLQP problem as triangular fuzzy number.

Step 2: Formulate the FFBLQP problem (1)-(3).

Step 3: Transform problem (1)-(3) to problem (4)-(6).

Step 4: Use the decomposition method to convert problem (4)-(6) into three crisp BLQP problems (7)-(9) by using the arithmetic operations on fully fuzzy.

Step 5: Calculate the individual fuzzy best and worst fuzzy solutions for each objective of problem (10) and problem (14) as in (11) and (15).

Step 6: Formulate the membership function of the fuzzy ULDM and fuzzy LLDM problems as in (12) and (16).

Step 7: Find the fuzzy solution of the fuzzy BLQP problem by solving the Tchebycheff problem as in (13) and (17). 
Operations Research and Applications : An International Journal (ORAJ), Vol.5, No.2, May 2018

Step 8: Formulate the membership function of the fuzzy BLQP problem as in (19), (20) and (21) after defined the value of fuzzy control decision variables $\left(\tilde{x}_{1}^{u}\right)$ and the maximum tolerance $\left(\tilde{t}_{1}\right)$.

Step 9: Formulate a Tchebycheff problem for BLQDM problem as in (18) to reach the fuzzy satisfactory solution.

Step 10: If $\tilde{\lambda}>0.5$, stop and the fuzzy compromise solution is obtained, $\tilde{\lambda}$ is the overall satisfaction for all $\mathrm{DM}_{\mathrm{s}}$, otherwise go to step8 after changing of the values $\tilde{t}_{1}$.

\section{NUMERICAL EXAMPLE}

To demonstrate the solution method for FFBLQP problem, let us consider the following example:

$$
\max _{\tilde{x}_{1}} \tilde{F}_{1}=(3,5,12) \otimes \tilde{x}_{1}^{2} \oplus(2,4,10) \otimes \tilde{x}_{2}^{2},
$$

where $\tilde{x}_{2}$ solves

$$
\begin{gathered}
\max _{\tilde{x}_{2}} \tilde{F}_{2}=(3,5,10) \otimes \tilde{x}_{1}^{2} \oplus(1,7,8) \otimes \tilde{x}_{2}^{2}, \\
\text { s.t. } \\
(4,5,6) \otimes \tilde{x}_{1}+(2,7,4) \otimes \tilde{x}_{2} \leq(4,10,20), \\
(2,3,4) \otimes \tilde{x}_{1}+(1,2,5) \otimes \tilde{x}_{2} \leq(2,5,8), \\
\tilde{x}=\left(\tilde{x}_{1}, \tilde{x}_{2}\right) \geq \tilde{0}
\end{gathered}
$$

where $\tilde{x}_{1}, \tilde{x}_{2}$ are triangular fuzzy numbers,

Assume that $\tilde{x}_{1}=\left(x_{1}, y_{1}, z_{1}\right), \tilde{x}_{2}=\left(x_{2}, y_{2}, z_{2}\right)$ and $\tilde{F}_{j}=\left(F_{j}^{L}, F_{j}^{m}, F_{j}^{u}\right)$,

$j=1,2$.

According to arithmetic operations of triangular fuzzy numbers, the FFBLQP problem (7)-(9) can be rewritten as:

FULDM: $\max _{x_{1}} F_{1}^{L}=3 x_{1}^{2}+2 x_{2}^{2}$,

$$
\begin{aligned}
& \max _{y_{1}} F_{1}^{m}=5 y_{1}^{2}+4 y_{2}^{2}, \\
& \max _{z_{1}} F_{1}^{u}=12 z_{1}^{2}+10 z_{2}^{2},
\end{aligned}
$$

where $\tilde{x}_{2}=\left(x_{2}, y_{2}, z_{2}\right)$ solves

FLLDM: $\max _{x_{2}} F_{2}^{L}=3 x_{1}^{2}+x_{2}^{2}$,

$$
\begin{gathered}
\max _{y_{2}} F_{2}^{m}=5 y_{1}^{2}+7 y_{2}^{2}, \\
\max _{z_{2}} F_{2}^{u}=10 z_{1}^{2}+8 z_{2}^{2}, \\
\text { s.t. }
\end{gathered}
$$


Operations Research and Applications : An International Journal (ORAJ), Vol.5, No.2, May 2018

$$
\begin{aligned}
& G=\left\{4 x_{1}+2 x_{2} \leq 4, \quad 2 x_{1}+x_{2} \leq 2,\right. \\
& 5 y_{1}+7 y_{2} \leq 10,3 y_{1}+2 y_{2} \leq 5 \text {, } \\
& 6 z_{1}+4 z_{2} \leq 20,4 z_{1}+5 z_{2} \leq 8, \\
& x_{1} \geq 0, \quad x_{2} \geq 0 \\
& y_{1}-x_{1} \geq 0 \quad, \quad y_{2}-x_{2} \geq 0 \\
& \left.z_{1}-y_{1} \geq 0 \quad, \quad z_{2}-y_{2} \geq 0\right\}
\end{aligned}
$$

where $\left(x_{1}, y_{1}, z_{1}\right)$ and $\left(x_{2}, y_{2}, z_{2}\right)$ are triangular fuzzy numbers.

First: The FULDM solves problem (10) as follows:

$$
\begin{aligned}
& \max _{\widetilde{x}_{1}} \widetilde{F}_{1}=\max _{\widetilde{x}_{1}}\left(F_{1}^{L}, F_{1}^{m}, F_{1}^{u}\right), \\
& \text { s.t. } \\
& \tilde{x}_{1} \in G .
\end{aligned}
$$

1- Find individual best fuzzy solution $\tilde{F}_{1}^{+}$and individual worst fuzzy solution $\tilde{F}_{1}^{-}$by solving (11), we get

\begin{tabular}{|l|l|l|l|l|}
\hline Upper level & $\tilde{x}_{1}=\left(x_{1}, y_{1}, z_{1}\right)$ & $\tilde{x}_{2}=\left(x_{2}, y_{2}, z_{2}\right)$ & $\tilde{F}_{1}^{+}=\max _{x_{1} \in G}\left(F_{1}^{L}, F_{1}^{m}, F_{1}^{u}\right)$ & $\tilde{F}_{1}^{-}=\max _{x_{1} \in G}\left(F_{1}^{L}, F_{1}^{m}, F_{1}^{u}\right)$ \\
\hline $\begin{array}{l}\text { Best fuzzy } \\
\text { solution }\end{array}$ & $(1,1.667,2)$ & $(0,0,0)$ & $\tilde{F}_{1}^{+}=(3,13889,48)$ & \\
\hline $\begin{array}{l}\text { Worst fuzzy } \\
\text { solution }\end{array}$ & $(0,0,0)$ & $(0,0,0)$ & & $\tilde{F}_{1}^{-}(0,0,0)$ \\
\hline
\end{tabular}

2- Use (12) to build the membership functions $\mu_{\widetilde{F}_{1}}\left[\tilde{F}_{1}(\tilde{x})\right]$ and solve (13) as follows:

\begin{tabular}{|l|l|l|}
\hline $\max \lambda_{1}^{L}$, & $\max \lambda_{1}^{m}$, & $\max \lambda_{1}^{u}$, \\
s.t. & s.t. & s.t. \\
$3 x_{1}^{2}+2 x_{2}^{2} \geq 3 \lambda_{1}^{L}$, & $5 y_{1}^{2}+4 y_{2}^{2} \geq 13.889 \lambda_{1}^{m}$, & $12 z_{1}^{2}+10 z_{2}^{2} \geq 48 \lambda_{1}^{u}$, \\
$4 x_{1}+2 x_{2} \leq 4$, & $5 y_{1}+7 y_{2} \leq 10 \quad$, & $6 z_{1}+4 z_{2} \leq 20$ \\
$2 x_{1}+x_{2} \leq 2,$, & $3 y_{1}+2 y_{2} \leq 5 \quad$, & $4 z_{1}+5 z_{2} \leq 8$ \\
$0 \leq \lambda_{1}^{L} \leq 1 \quad$. & $0 \leq \lambda_{1}^{m} \leq 1$ & $0 \leq \lambda_{1}^{u} \leq 1$ \\
\hline
\end{tabular}

whose solution is:

$\tilde{x}_{1}^{u}=(0.251,1.667,2), \tilde{x}_{2}^{u}=(1.253,0,0), \tilde{F}_{1}^{u}=(3.329,13.894,48)$

$\tilde{\lambda}_{1}=(1,1,1)$ and $\tilde{F}_{1}^{L}=(4.501,8.168,26.499)$

Second: In the same way, the FLLDM solves problem (14) as follows:

1- Find individual best fuzzy solutions $\tilde{F}_{2}^{+}$and individual worst fuzzy solutions $\tilde{F}_{2}^{-}$by solving (15), we get 
Operations Research and Applications : An International Journal (ORAJ), Vol.5, No.2, May 2018

\begin{tabular}{|l|l|l|l|l|}
\hline Lower level & $\tilde{x}_{1}=\left(x_{1}, y_{1}, z_{1}\right)$ & $\tilde{x}_{2}=\left(x_{2}, y_{2}, z_{2}\right)$ & $\tilde{F}_{2}^{+}=\max _{x_{2} \in G}\left(F_{2}^{L}, F_{2}^{m}, F_{2}^{u}\right)$ & $\tilde{F}_{2}^{-}=\max _{x_{2} \in G}\left(F_{2}^{L}, F_{2}^{m}, F_{21}^{u}\right)$ \\
\hline $\begin{array}{l}\text { Best fuzzy } \\
\text { solution }\end{array}$ & $(0,0,0)$ & $(1.429,1.429,1.6)$ & $(2.042,14.286,20)$ & \\
\hline $\begin{array}{l}\text { Worst fuzzy } \\
\text { solution }\end{array}$ & $(0,0,0)$ & $(0,0,0)$ & & $(0,0,0)$ \\
\hline
\end{tabular}

2- By using (16), build the membership functions $\mu_{\widetilde{F}_{2}}\left[\tilde{F}_{2}(\tilde{x})\right]$ and solve (17) we get: $\tilde{x}_{1}^{L}=(0.119,0,1.44), \tilde{x}_{2}^{L}=(1.493,1.429,0.402), \tilde{\lambda}_{2}=(1,1,1)$, $\tilde{F}_{2}^{L}=(2.272,14.294,22.029), \tilde{F}_{2}^{u}=(1.759,13.894,40)$

Third: In order to generate the fuzzy satisfactory solution,

1- Assume the FULDM' fuzzy control decision $\tilde{x}_{1}^{u}$ is around $(0.251,1.667,2)$ with the tolerance $=0.2$.

2- By (19), (20) and (21) and from membership functions, $\mu^{\prime}\left(\tilde{x}_{1}\right), \mu_{\tilde{F}_{1}}^{\prime}\left[\tilde{F}_{1}(\tilde{x})\right], \mu_{\tilde{F}_{2}}^{\prime}\left[\tilde{F}_{2}(\tilde{x})\right]$, the FLLDM solves the following Tchebycheff problem (18) as:

\begin{tabular}{|l|l|l|}
\hline $\max \lambda^{L}$, & $\max \lambda^{m}$, & $\max \lambda^{u}$, \\
s.t. & s.t. & s.t. \\
$x_{1}+0.2 \lambda_{1}^{L} \leq 0.451$, & $y_{1}+0.2 \lambda^{m} \leq 1.867$, & $z_{1}+0.2 \lambda^{u} \leq 2.2$, \\
$x_{1}-0.2 \lambda^{L} \geq 0.051$, & $y_{1}-0.2 \lambda^{m} \geq 1.467$, & $z_{1}-0.2 \lambda^{u} \geq 1.8 \quad$, \\
$3 x_{1}^{2}+2 x_{2}^{2}+1.172 \lambda^{L} \geq 4.501$, & $5 y_{1}^{2}+4 y_{2}^{2}-5.726 \lambda^{m} \geq 8.168$, & $12 z_{1}^{2}+10 z_{2}^{2}-21.501 \lambda^{u} \geq 26.499$, \\
$3 x_{1}^{2}+x_{2}^{2}-0.513 \lambda^{L} \geq 1.759$, & $5 y_{1}^{2}+7 y_{2}^{2}-0.4 \lambda^{m} \geq 13.894$, & $10 z_{1}^{2}+8 z_{2}^{2}+17.971 \lambda^{u} \geq 40$, \\
$0 \leq \lambda^{L} \leq 1$ & $0 \leq \lambda^{m} \leq 1$. & $0 \leq \lambda^{u} \leq 1$ \\
& & \\
\hline
\end{tabular}

Therefore, the compromise fuzzy solutions are

$\tilde{x}_{1}=(0.251,1.667,2), \quad \tilde{x}_{2}=(1.261,2.471,2.807)$,

$\tilde{F}_{1}=(3.369,38.318,126.792), \quad \tilde{F}_{2}=(1.779,56.635,463.034)$,

and $\tilde{\lambda}=\left(\lambda^{L}, \lambda^{m}, \lambda^{u}\right)=(1,1,1)$ (overall satisfaction for both $\left.\mathrm{DM}_{\mathrm{s}}\right)$.

\section{CONCLUSION}

In this paper, a fully fuzzy bi-level quadratic programming problem in which all the coefficients and decision variables are fuzzy numbers is introduced. In order to obtain a fuzzy optimal solution of the FFBLQP problem, the concepts of tolerance membership functions at each level to develop a fuzzy max-min decision model for generating fuzzy satisfactory solution for FFBLQP problem. Then the fully fuzzy bi-level quadratic programming can be converted into a deterministic bi-level programming problem by using the bound and decomposition method. Also, a new algorithm is based on the fuzzy decision approach, bound and decomposition method 
Operations Research and Applications : An International Journal (ORAJ), Vol.5, No.2, May 2018

are introduced to obtain the fuzzy satisfactory solution for FFBLQP problem. Finally, an illustrative numerical example has been given to clarify the proposed solution method.

\section{REFERENCES}

[1] H.S. Shih, Y.J. Lai and L.E. Stanley., Fuzzy Approach for Multi-Level Programming Problems., Computers and Operations Research, 23(1), 73-91, (1996).

[2] X. Shi and H. Xia., Interactive Bi-Level Multi -Objective Decision Making., Journal of Operational Research Society, 48, 943-949, (1997).

[3] M.A. Abo-Sinna and A.H. Amer., Extension of TOPSIS for Multi-Objective Large-Scale NonLinear Programming Problems., Appl. Math. Comput., 162, 243-256, (2000).

[4] M. A. Abo-Sinna., A Bi-level Non-Linear Multi-Objective Decision Making Under Fuzziness., J. Oper. Res. Soc. India (OPSEARCH), 38(5), 484-495, (2001).

[5] M.S. Osman, M.A.Abo-Sinna, A.H.Amer and O.E.Eman., A Multi-Level Non-Linear MultiObjective Under Fuzziness., Applied Mathematics and Computations, 153, 239-252, (2004).

[6] A.H. Amer and M.A. Abo-Sinna., An Interactive Dynamic Fuzzy Goal Programming for Bi-Level Multi-Objective Linear Fractional Programming Problems., Journal of Advances in Mathematics, 12 (12), 6991-7007 (2017).

[7] M.A. Abo. Sinna and A.H. Amer., TOPSIS Approach for Solving Bi-Level Fractional MODM Problems., Journal of Advances in Mathematic, 13 (4), 7353-7370, (2018).

[8] A. H. Amer., Implementation of the $\in$-Constraint Method in Special Class of Multi-Objective Fuzzy Bi-Level Non-Linear Problems., Pak.J. Stat. Oper. Res., vol. XIII, no.4, 739-756,(2017).

[9] A.H. Amer., On the Karush-Kuhn-Tucker Reformulation of Bi-Level Geometric Programming Problem with an Interval Coefficients as Multiple Parameters., Journal of Progressive Research in Mathematics (JPRM), 11(2), 1566-1577, (2017).

[10] J. F. Bard., Practical Bi-Level Optimization: Algorithms and Applications., Kluwer Academic Publishers, Dordrecht, the Nether-Lands, (1998).

[11] B. Colson, P. Marcotte and G. Savard., An Overview of Bi-Level Optimization., Annals of Operations Research, vol. 153, pp. 235-256, (2007).

[12] F. Gzara., A Cutting Plane Approach for Bi-Level Hazardous Material Transport Network Design., Operations Research Letters, vol.41, no.1, pp.40-46, (2013).

[13] T.Kis and A. Kovacs., Exact solution Approaches for Bi-Level Lot-Sizing., Eur. J. Oper. Res., 226 (2), 237-245, (2013).

[14] M. W. Xu and J.J. ye., A smoothing Augmented Lagrangian Method for Solving Simple Bi-Level Programs., Comput. Optim. Appl., 59(1), 353-377, (2014).

[15] V. Kalashnikov, T.1. Matis, J.F.C. Vallejo and S.V. Kavun., Bi-Level Programming Equilibrium and Combinatorial Problems with Applications to Engineering., Math. Probl. Eng., vol. 2015, 14, (2015).

[16] L.A. Zadeh., Fuzzy Sets., Information and Computation, vol. 8, no. 3,pp.338-353, (1965).

[17] H. Tanaka, T. Okuda and K. Asai., On Fuzzy Mathematical Programming., Journal of Cybernetics, 3, 37-46, (1974).

[18] R.E. Bellman and L.A. Zadeh., Decision Making in a Fuzzy Environment., Management Science, 17, 141-164, (1970).

[19] H. J. Zimmermann., Fuzzy Programming and Linear Programming with Several Objective Functions., Fuzzy Sets and Systems, 1,45-55, (1978). 
Operations Research and Applications : An International Journal (ORAJ), Vol.5, No.2, May 2018

[20] M. Sakawa and H. Yano., Interactive Decision Making for Multi-Objective Non-Linear Programming Problems with Fuzzy Parameters., Fuzzy Sets and Syst., 29(3), 315-326, (1989).

[21] M. Sakawa, I. Nishizaki and Y. Uemura., Interactive Fuzzy Programming for multi-Level Linear Programming Problems with Fuzzy Parameters., Fuzzy Set and Syst., 109 (1), 3-19, (2000).

[22] M. Sakawa, I. Nishizaki and Y. Uemura., Interactive Fuzzy Programming for Two-Level Linear Fractional Programming Problems with Fuzzy Parameters., Fuzzy Sets and Syst., 115, 93-103, (2000)

[23] M. Sakawa, I. Nishizaki and Y. Uemura., Interactive Fuzzy Programming for Two-Level Linear and Linear Fractional Production and Assignment Problems: A Case Study., European Journal of Oper. Res., 135, 142- 157, (2001).

[24] T.S. Liou and M.J. Wang., Ranking Fuzzy numbers with Integral Value., Fuzzy Sets and Systems, vol. 50, no.3,pp.247-255, (1992).

[25] T. Allahviranloo, F. H. Lotfi, M.K. Kiasary, N.A. Kiani and L. Alizadeh., Solving Fully Fuzzy Linear Programming Problem by the Ranking Function., Applied Mathematical Sciences, 2, 19-32, (2008).

[26] F.H. Lotfi, T. Allahviranloo, M.A. Jondabeha and L. Alizadeh., Solving a Fully Fuzzy Linear Programming Using Lexicography Method and Fuzzy Approximate Solution., Applied Mathematical Modeling, 33,3151-3156, (2009).

[27] A. Kumar, J. Kaur and P. Singh., Solving Fully Fuzzy Linear Programming Problems with Inequality Constraints., International Journal of Physical and Mathematical Sciences, 1,6-18, (2010).

[28] A. Kumar, J. Kaur and P. Singh., Fuzzy Optimal Solution of Fully Fuzzy Linear Programming Problems with Inequality Constraints., International Journal of Applied Mathematics and Computer Sciences, 6, $37-41,(2010)$.

[29] A. Kumar, J. Kaur And P. Singh., A New Method for Solving Fully Fuzzy Linear Programming Problems., Applied Mathematical Modeling, 35, 817 - 823, (2011).

[30] M. Deb And P. K. De., Optimal Solution of a Fully Fuzzy Linear Fractional Programming Problem by Using Graded Mean Integration Representation Method., An International Journal of Applications and Applied Mathematics, 10(1), 571 - 587, June (2015).

[31] R. Ezzati, E. Khorram and R. Enayati., A New Algorithm to Solve Fully Fuzzy Linear Programming Problems Using the MOLP Problems., Applied Mathematical Modeling, 39, 3185 - 3193, (2015).

[32] H. S. Najafi, S. A. Edalatpanah and H. Dutta., A Non - Linear Model for Fully Fuzzy Linear Programming with Fully Unrestricted Variables and Parameters., Alexandria Engineering Journal, $55,2589-2595,(2016)$.

[33] W. I. Gabr., Quadratic and Non - Linear Programming Problems Solving and Analysis in Fully Environment., Alexandria Engineering Journal, 54, 457 - 472, (2015).

[34] A. Ren., Research Article, Solving the Fully Fuzzy Bi - Level Linear Programming Problem through Deviation Degree Measures and a Ranking Function Method., Mathematical Problems in Engineering, Volume 2016, Article ID 7069804, 11 Pages, (2016).

[35] A. Ren., Research Article, A Novel Method for Solving the Fully Fuzzy Bi - Level Linear Programming Problem., Mathematical Problems in Engineering, Volume 2015, Article ID 280380, 11 Pages, (2015).

[36] A. Ren, Y. Wang and X. Xue., Interactive Programming Approach for Solving the Fully Fuzzy Bi - Level Linear Programming Problem., Knowledge - Based Systems, 99, 103 - 111, (2016). 
Operations Research and Applications : An International Journal (ORAJ), Vol.5, No.2, May 2018

[37] N. Safaei and M. Saraj., A New Method for Solving Fully Fuzzy Linear Bi - Level Programming Problems., International Journal of Applied Operational Research, vol. 4, no.1, pp. 39 - 46, (2014).

[38] H. J. Zimmermann., Fuzzy Sets., Decision Making and Expert Systems, Kluwer Academic, Boston, (1987).

[39] D. Dubois and H. Prade., Fuzzy Sets and Systems: Theory and Applications, Academic Press, New York, (1980). 